\title{
Physical design of magnetic shielding for LEReC cooling section
}

\author{
S. Seletskiy, A. Fedotov, D. Gassner, \\ D. Kayran, G. Mahler, W. Meng, P. Thieberger
}

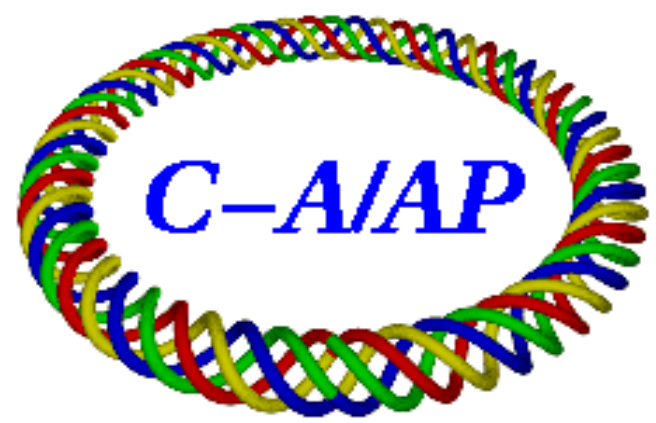

Collider-Accelerator Department Brookhaven National Laboratory Upton, NY 11973

\section{U.S. Department of Energy Office of Science, Office of Nuclear Physics}

Notice: This document has been authorized by employees of Brookhaven Science Associates, LLC under Contract No. DE-SC0012704 with the U.S. Department of Energy. The United States Government retains a nonexclusive, paid-up, irrevocable, world-wide license to publish or reproduce the published form of this document, or allow others to do so, for United States Government purposes. 


\title{
Physical Design of Magnetic Shielding for LEReC Cooling Section
}

April 5, 2016

\author{
S. Seletskiy, A. Fedotov, D. Gassner, D. Kayran, G. Mahler, W. Meng, P. Thieberger
}

\section{Introduction}

The transverse angle of the electron beam trajectory in LEReC cooling section (CS) must be much smaller than $\theta_{\max }=100$ urad. Since the smallest e-beam energy is going to be $1.6 \mathrm{MeV}$ $(B \rho=68.3 \mathrm{G} \cdot \mathrm{m})$, the ambient transverse magnetic field must be suppressed to:

$$
B_{\perp} \ll \frac{B \rho \theta_{\max }}{L}=2.3 \mathrm{mG}
$$

where $L=3 \mathrm{~m}$ is solenoid-to-solenoid distance in the cooling section.

The maximum ambient field in the RHIC tunnel along the cooling section was measured to be $0.52 \mathrm{G}$ (see Appendix A for details). Assuming that $1 \mathrm{mG}$ residual transverse field is low enough we find the required attenuation factor $S$ to be 520 .

It was Fermilab Electron Cooler experience [1] that due to mechanical joints of the shields actual attenuation of magnetic shielding can be almost two times smaller than the designed one. Therefore, we suggest adding a safety factor of two to our model making design $S=$ 1040.

The goal of this note is to set basic parameters for the magnetic shielding of LEReC CS with required design attenuation.

\section{Comparing analytic formulas to 3D simulations}

The systematic studies of shielding of the magnetic fields with cylindrical shells of high permeability material [2] were summarized in the form of simple analytic formulas [3, 4].

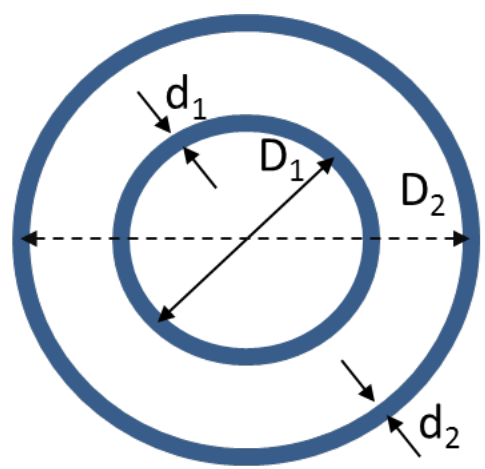

Figure 1: Schematic of 2 layer shielding with cylindrical shells (picture plane is normal to the axis of the cylinders). 
The attenuation factor of the long cylinder with diameter $D$, thickness $d$ and magnetic permeability $\mu$ is:

$$
S=1+\mu \frac{d}{D}
$$

For two cylindrical layers (see Fig. 1 for notations) with respective attenuations $S_{1}$ and $S_{2}$ :

$$
S=S_{1} S_{2}\left(1-\left(\frac{D_{1}}{D_{2}}\right)^{2}\right)+S_{1}+S_{2}+1
$$

The general formula for $N$ layers is:

$$
S=1+\sum_{n=1}^{N} S_{n}+S_{N} \prod_{n=1}^{N-1} S_{n}\left[1-\left(\frac{D_{n}}{D_{n+1}}\right)^{2}\right]
$$

We applied (1)-(3) to 4 different shielding setups and compared obtained attenuations with results of 3D simulations. Results of the comparison are given in Table 1.

\begin{tabular}{|l|l|l|l|l|l|l|}
\hline & \multicolumn{1}{|c|}{$\mu$} & \multicolumn{1}{|c|}{ N layers } & \multicolumn{1}{|c|}{$\mathrm{D}_{1,2,3}, \mathrm{~mm}$} & \multicolumn{1}{c|}{$\mathrm{d}, \mathrm{mm}$} & \multicolumn{1}{|c|}{$\mathrm{S}_{\text {formula }}$} & \multicolumn{1}{c|}{$\mathrm{S}_{\text {simulation }}$} \\
\hline LEReC 1 & 15000 & 2 & 300,400 & 1 & $\mathbf{9 5 0}$ & $\mathbf{8 7 7}$ \\
\hline LEReC 2 & 11000 & 2 & 300,400 & 1 & $\mathbf{5 3 7}$ & $\mathbf{4 8 5}$ \\
\hline Fermilab 1 & 15000 & 3 & $219,233.4,267.2$ & 1 & $\mathbf{7 5 3 8}$ & $\mathbf{7 4 0 0}$ \\
\hline Fermilab 2 & 11000 & 3 & $219,241.2,266.6$ & 1 & $\mathbf{3 3 5 6}$ & $\mathbf{3 0 0 0}$ \\
\hline
\end{tabular}

Table 1: Comparison of $S$ found from analytic formulas (column 6 ) and $S$ found from 3D simulations (column 7).The second and the third rows correspond to Opera [5] simulations of two possible LEReC setups [6]. The fourth and the fifth rows correspond to the test and the final Fermilab setups $[1,7]$.

As one can see, the formulas agree with simulations with $\sim 10 \%$ precision. Therefore, we will use these formulas for initial optimization of the design parameters for LEReC CS shielding.

\section{Physical design of LEReC CS shielding}

According to (1) it is beneficial to make the diameter of the first layer as small as possible. The diameter of the vacuum chamber is $5^{\prime \prime}$. Therefore, the radius of the first shielding layer will be 5 " as well. There are bellows and flanges of 7" OD at 17.5" downstream from the face of each 
solenoid and BPM buttons located about 9.5" downstream of the face of each solenoid sticking out even more. Thus, the first layer of shielding can start only at $17.5^{\prime \prime}$ downstream of the solenoid face. It can go uninterrupted basically right to the entrance of the next solenoid.

We choose our shields to be $1 \mathrm{~mm}$ thick. Such shields will keep their form through reannealing process required after mechanical work on the shields is finished to improve the permeability of the material. From Fermilab experience $[1,7,8]$ we expect to get $\mu=11000$.

Optimization of the radius of the second shielding layer is demonstrated in Fig. 2.

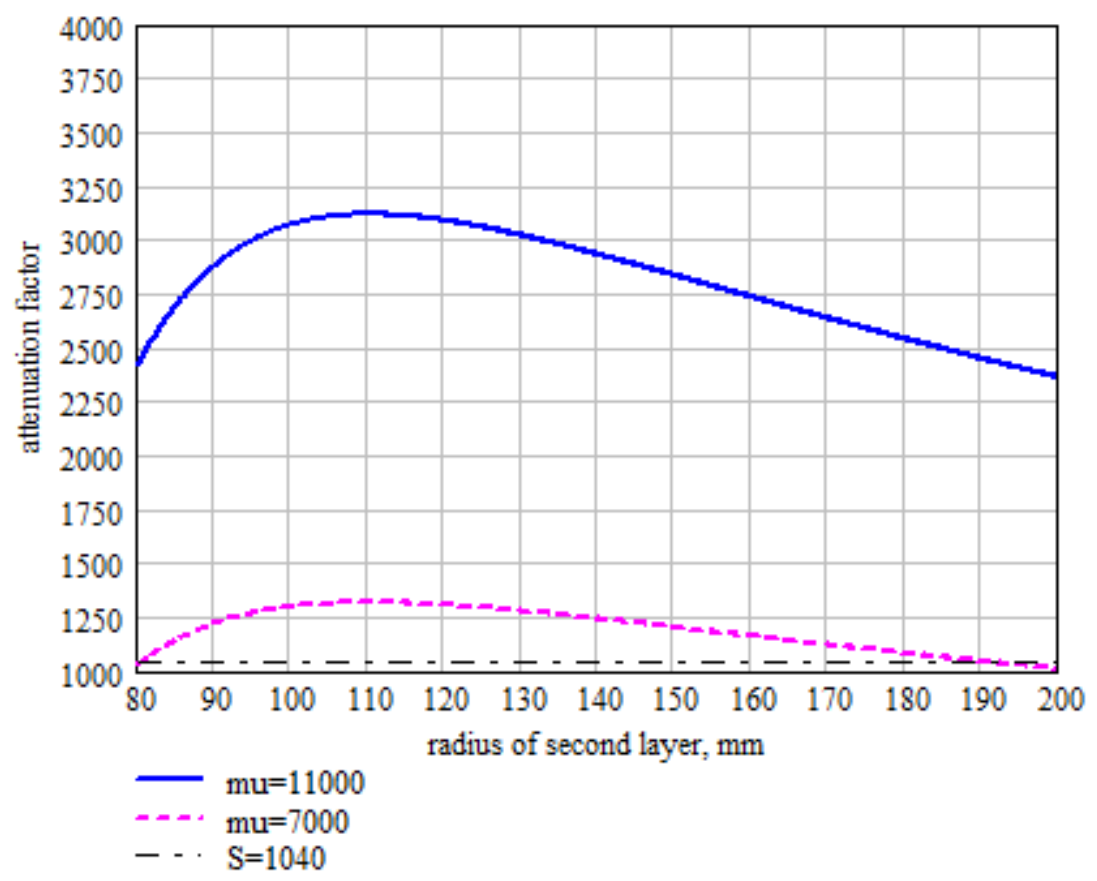

Figure 2: Attenuation is optimized for second layer radius of $110 \mathrm{~mm}$ but for convenience we choose this radius to be $150 \mathrm{~mm}$. Solid blue line shows $S$ for nominal $\mu=11000$. Dashed magenta line shows $\mu=7000$ case.

The second layer of shielding would maximize attenuation for $R_{2}=110 \mathrm{~mm}$, yet for the reasons of design convenience we choose $R_{2}=150 \mathrm{~mm}$. Such radius of the second layer of shielding still gives theoretical attenuation factor of 2800 , which is much better than our requirements. As a matter of fact, for chosen shielding, as Fig. 2 shows, one can get adequate attenuation even if $\mu$ degrades to 7000 .

The $15 \mathrm{~cm}$ radius shield can start at just 1 " away from the face of the solenoid.

The first $445 \mathrm{~mm}$ of each solenoid-to-solenoid $3 \mathrm{~m}$ drift will be covered by a single $15 \mathrm{~cm}$ radius layer of shielding. Thus, according to (1) the attenuation in this region will be about 38. As it will be shown in the next section such design of the CS shielding is still acceptable when one considers compensation of acquired transverse angles with correctors (located in each solenoid). 


\section{CS shielding design and e-beam trajectory angles}

The design discussed in the previous chapter is schematically shown in Fig. 3.

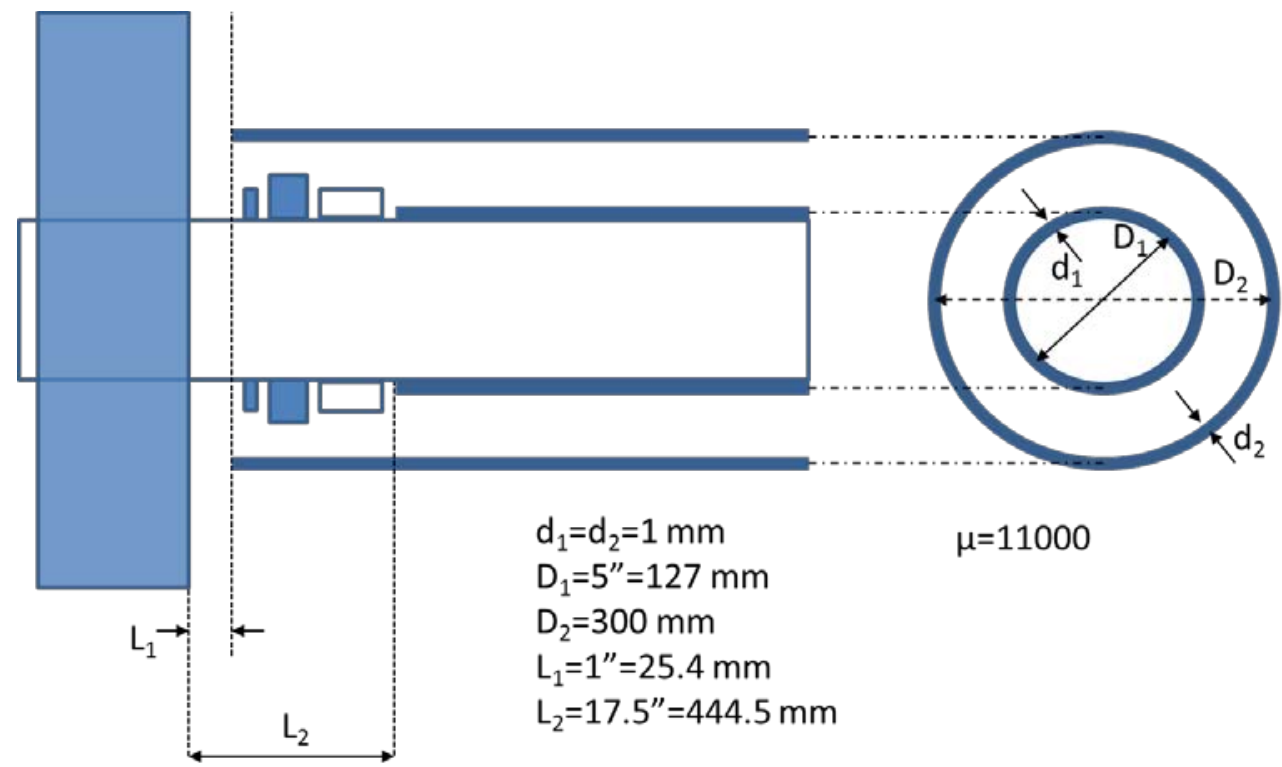

Figure 3: Schematics of the proposed magnetic shielding of LEReC CS.

We performed Opera 3D simulations for such shielding setup (see Fig. 4) assuming the external transverse field to be $0.52 \mathrm{G}$ homogeneously distributed and pointing in $\mathrm{x}$-direction.

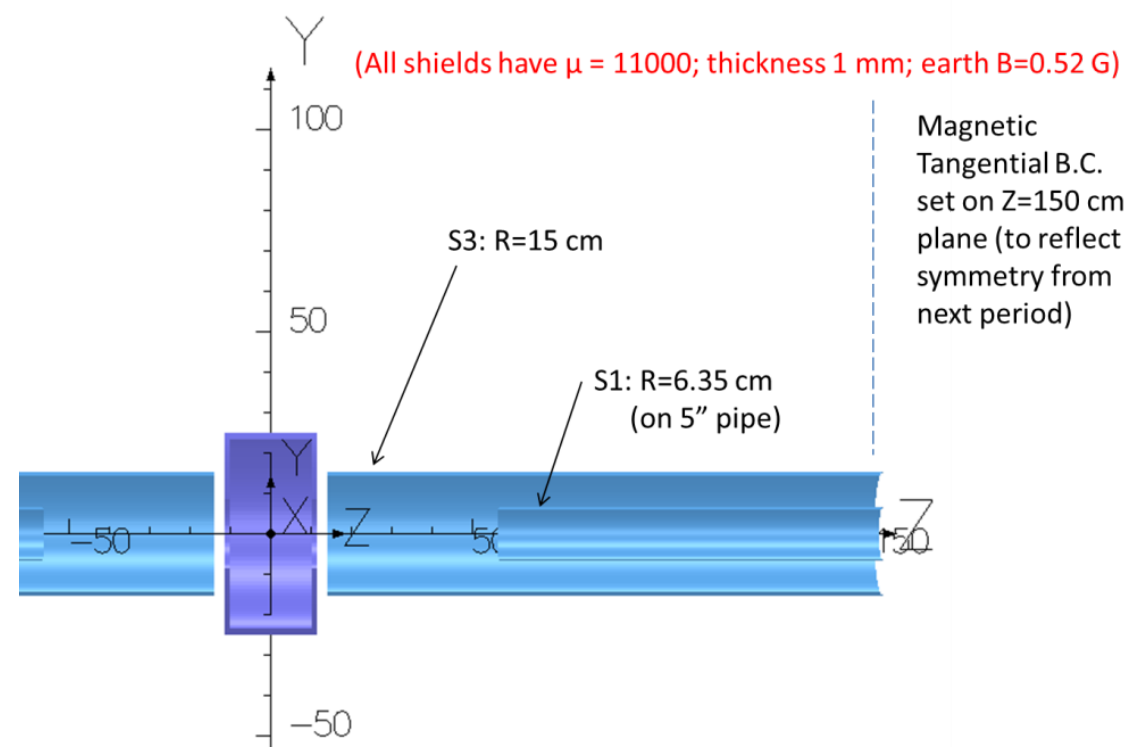

Figure 4: Setup for 3D shielding simulations. 
To account for the required safety factor of 2 we assume that real life field in the cooling section can be twice as high as the field found in simulation as Fig. 5 demonstrates.

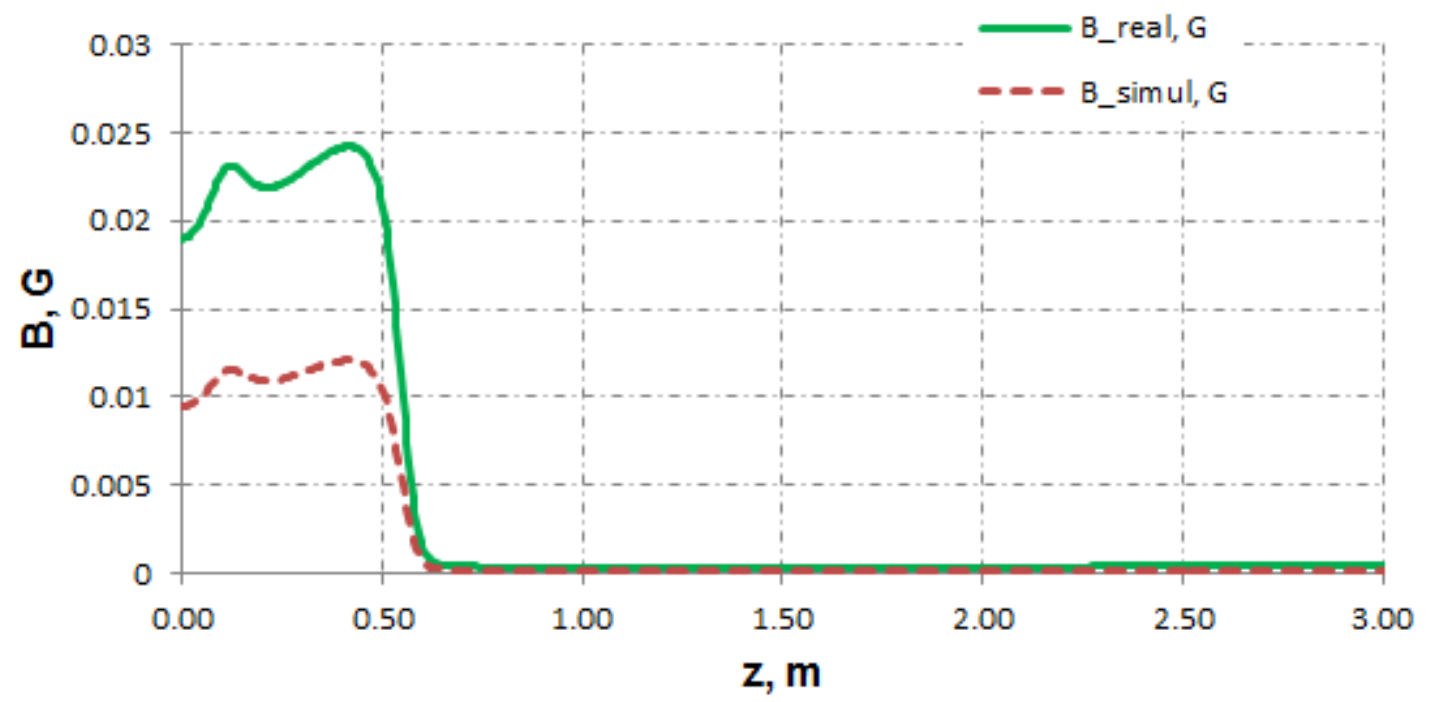

Figure 5: Simulated and "real" (multiplied by 2) residual magnetic field in the cooling section.

Finally, we simulate the trajectory of the $1.6 \mathrm{MeV}$ (kinetic energy) beam traveling for $3 \mathrm{~m}$ in the transverse field shown in Fig. 5. As one can see (Fig. 6), the e-beam trajectory angle in such field satisfies LEReC requirements everywhere but in the first $20 \mathrm{~cm}$ from the solenoid center (which is OK, since this distance is lost for cooling anyway due to the presence of strong solenoidal field).

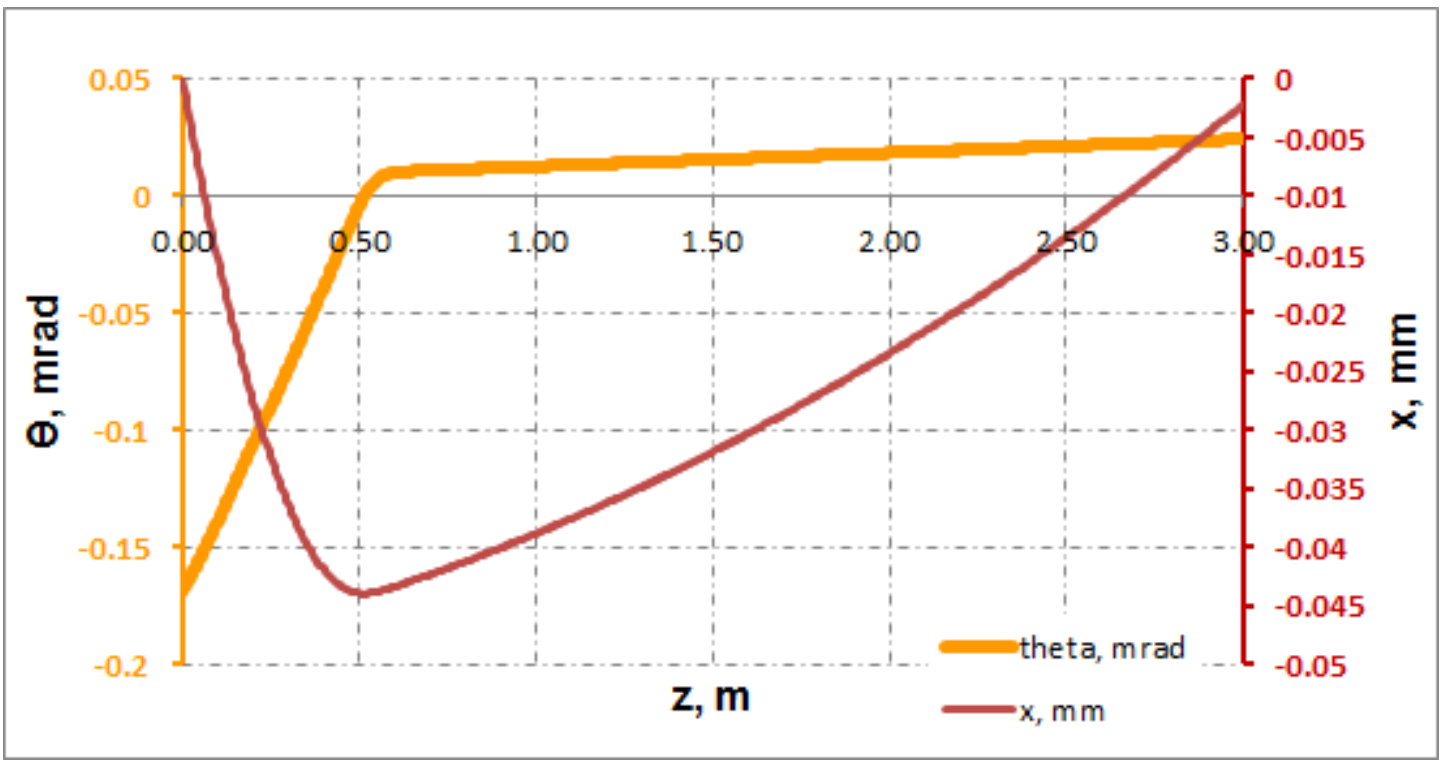

Figure 6: Beam angle (orange) and trajectory (red) in the "real" field shown in Fig. 5. 
In our beam trajectory simulations we compensate the beam deflection through the drift by applying 0.17 mrad kick in transverse corrector located inside the solenoid. We also assume a simple procedure for tuning beam trajectory - we use CS trajectory corrector in every solenoid to zero beam displacement in the next respective BPM.

The nominal strength of CS transverse correctors, which are designed to compensate possible misalignments of solenoids, is $10 \mathrm{G} \cdot \mathrm{cm}$ [9] while its measured strength is $8.5 \mathrm{G} \cdot \mathrm{cm}$ for $0.8 \mathrm{~A}$ current [10]. Thus, for nominal settings such corrector can produce at least 1.25 mrad kick, which is 7 times higher than the kick required for compensating the effect of residual transverse field.

Finally, it is important to notice that the longitudinal component of the ambient field (see Appendix A for details) will not be well shielded by cylindrical shells. Nonetheless, this field is so small (less than $0.35 \mathrm{G}$ ) that it has negligible effect on the angle of beam trajectory.

\section{Conclusion}

We considered physical design of magnetic shielding of LEReC cooling section.

The schematic of this design along with the list of its basic parameters is shown in Fig. 3.

We are planning to use 2 layers of $1 \mathrm{~mm}$ thick cylindrical mu-metal shields with $\mu=11000$.

The radius of the first layer sitting on top of vacuum chamber is $63.5 \mathrm{~mm}$. The second layer radius is $150 \mathrm{~mm}$.

Such shielding guarantees adequate transverse angles of electron beam trajectory in the CS.

\section{References}

[1] A.C. Crawford et al., Field Measurement in the Fermilab Electron Cooling Solenoid Prototype, FERMILAB-TM-2224, 2003.

[2] A.P. Wills, On the magnetic shielding of tri-lamellar spherical and cylindrical shells, Phys. Rev., Vol. 9, 1899.

[3] A. K. Thomas, IEEE Trans. Electromagn. Compat. 10, 142 (1968).

[4] A. Mager, IEEE Trans. Magn. MAG-6, 67 (1970).

[5] http://operafea.com/

[6] W. Meng, Magnetic Shielding Study, April 30, 2015.

[7] J. Leibfritz et al., Fermilab Electron Cooling Project, Engineering Aspects of Cooling Section, FERMILAB-Conf-01/163 E901, 2001.

[8] T.K. Kroc, C.W. Schmidt, A. Shemyakin, Magnetic Shielding of an Electron Beamline in a Hadron Accelerator Enclosure, Proceedings of PAC05, Knoxville, Tennessee, 2005.

[9] G. Mahler, Statement of Work for Low Energy RHIC Electron Cooling Compensating Solenoid Magnet Assemblies, 010606318 SOW-01, December 21, 2014.

[10] A. Jain, Results of Magnetic Measurements in LEReC Solenoids, November 24, 2015. 


\section{Appendix A}

\section{Measurements of Ambient Magnetic Field in LEReC Cooling Section and Transport Line}

We performed two sets of measurements of ambient magnetic field in RHIC tunnel where LEReC transport line and cooling section will be installed.

The first measurement was done with RHIC magnets turned off. The second measurement was performed $\sim 1.5$ months later with RHIC magnets turned on at injection energy. Both measurements were done with Lakeshore 3D Hall probe (see Fig. A1).

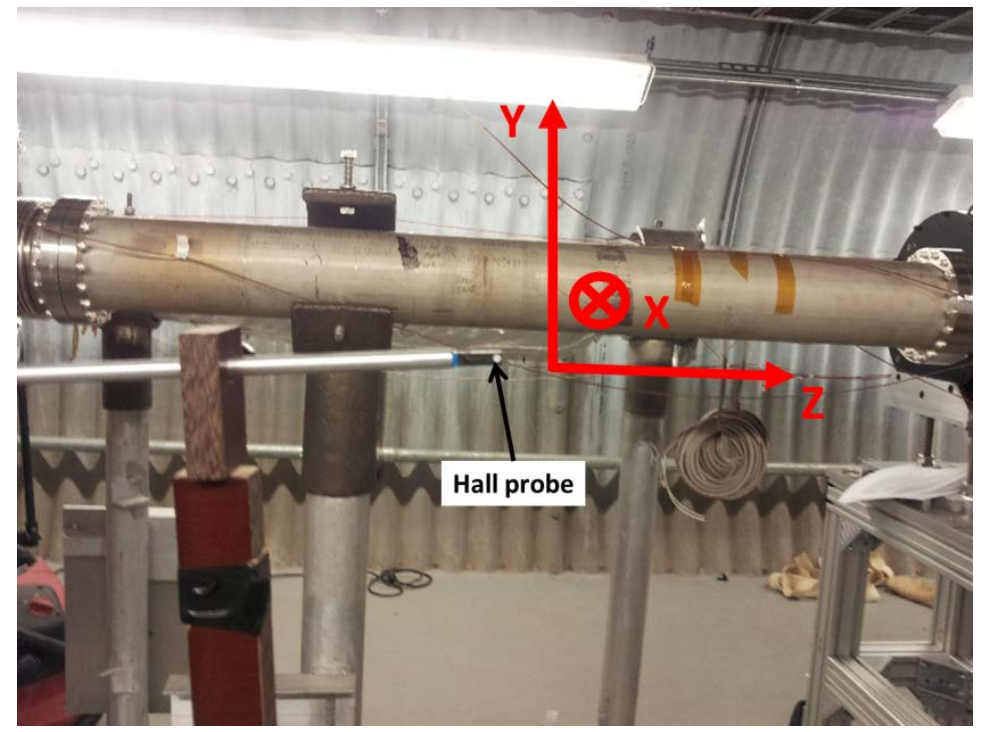

Figure A1: Measurement setup.

The results of the measurements for the transport line are shown in Fig. A2. Cooling section measurement results are shown in Fig. A3.

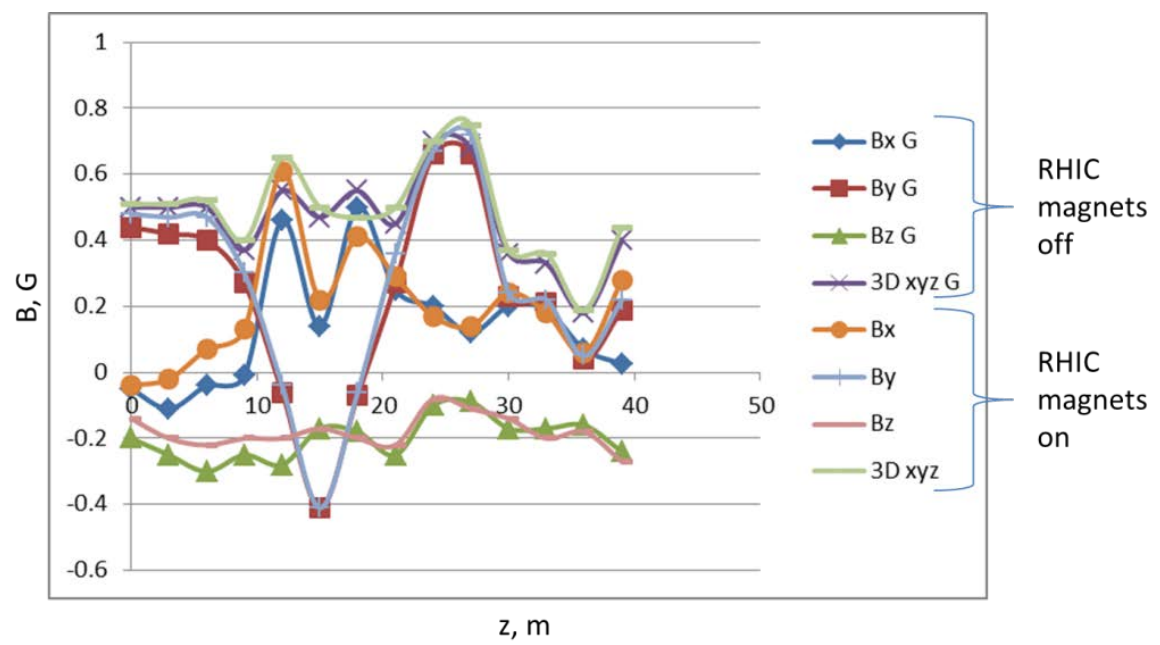

Figure A2: Ambient magnetic field in LEReC transport line. 

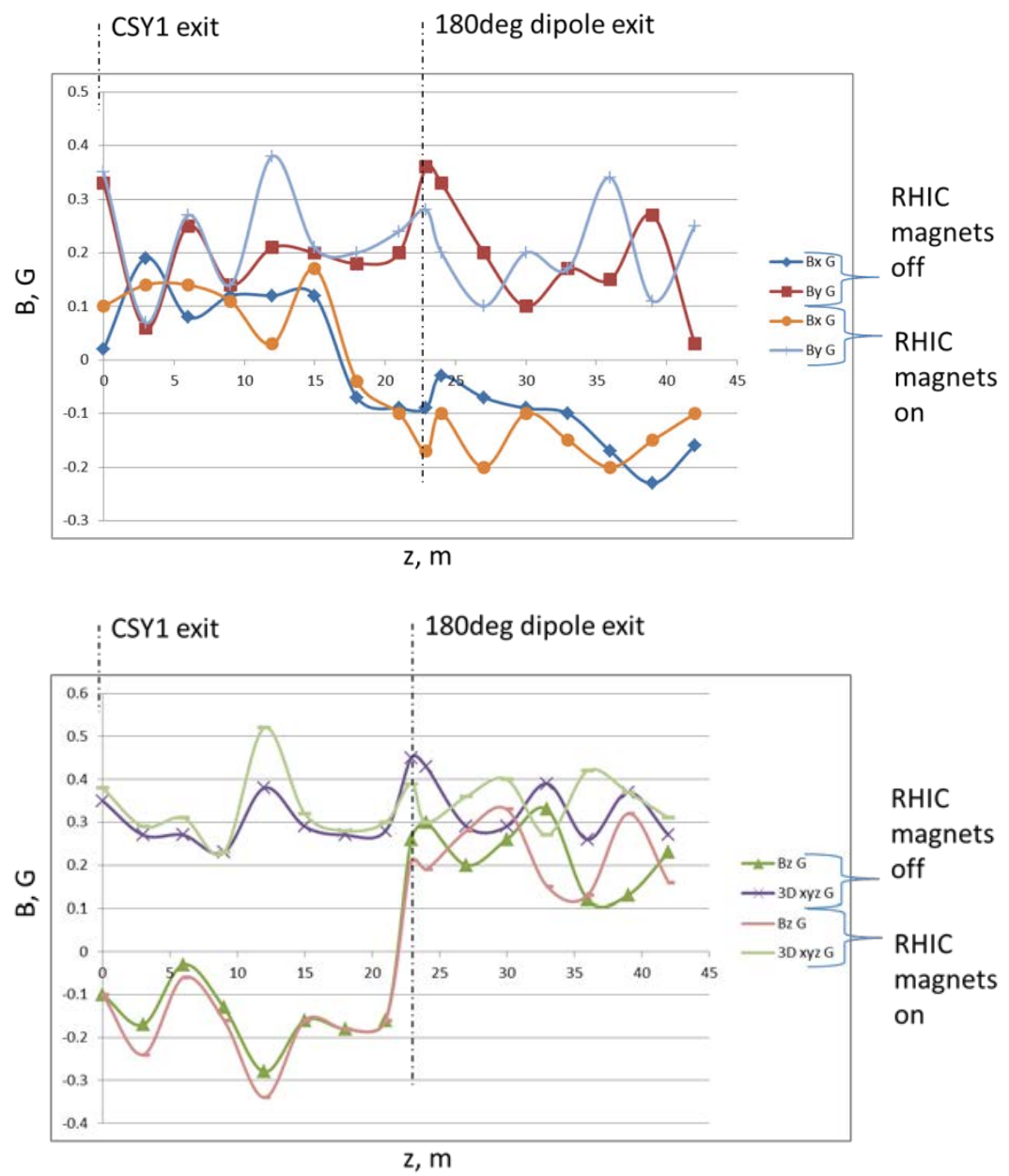

Figure A3: Ambient magnetic field in LEReC CS. $X$ and $Z$ field components change their sign as we pass 180 degree bend because the probe is getting rotated by 180 degrees.

As one can see there are no substantial changes in field readings between RHIC magnets being switched off and on. The maximum field in the CS is $0.52 \mathrm{G}$ and although it is not completely transverse we are using this value as a maximum possible transverse field in our simulations.

Figure A3 shows that the longitudinal component of the ambient field varies along the cooling section but never exceeds $0.35 \mathrm{G}$. As our simulations show, this value is too small to cause any noticeable effect on transverse angles of beam trajectory.

Finally, Fig. A4 summarizes the results of the ambient field measurement throughout the whole LEReC in a convenient form. 


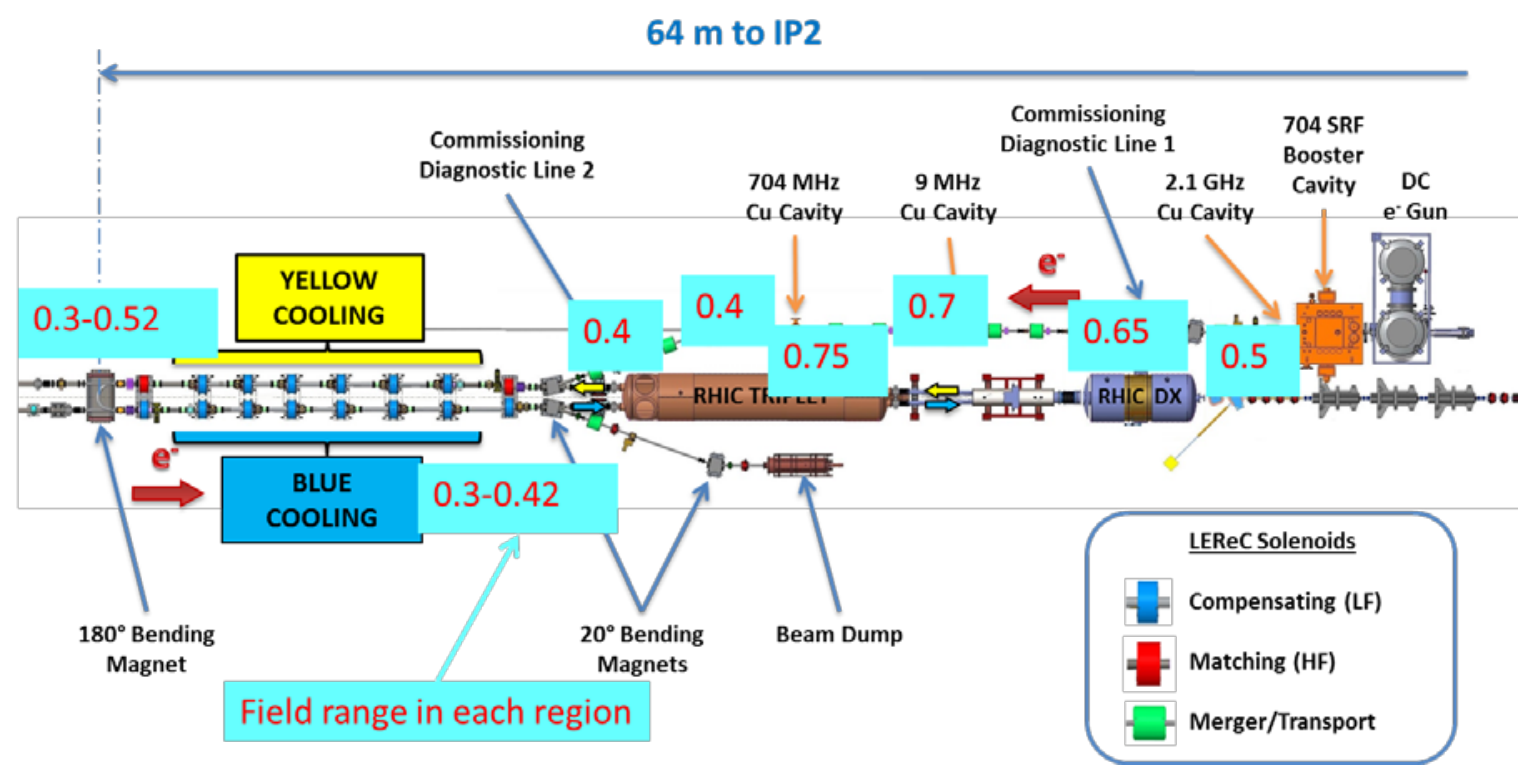

Figure A4: Summary of the ambient magnetic field measurements in RHIC tunnel. 